\title{
miR-29b overexpression induces cochlear hair cell apoptosis through the regulation of SIRT1/PGC-1 $\alpha$ signaling: Implications for age-related hearing loss
}

\author{
TAO XUE $^{1 *}$, LI WEI $^{2 *}$, DING-JUN ZHA ${ }^{1}$, JIAN-HUA QIU $^{1}$, FU-QUAN CHEN $^{1}$, LI QIAO $^{1}$ and YANG QIU ${ }^{1}$ \\ Departments of ${ }^{1}$ Otolaryngology, and ${ }^{2}$ Obstetrics and Gynecology, Xijing Hospital, \\ The Fourth Military Medical University, Xi'an, Shaanxi 710003, P.R. China
}

Received December 21, 2015; Accepted August 25, 2016

DOI: $10.3892 /$ ijmm.2016.2735

\begin{abstract}
It has been reported that the degeneration of cochlear hair cells is the typical cause of presbycusis (or age-related hearing loss). However, the molecular mechanisms that mediate cochlear hair cell apoptosis are not yet fully understood and there is no effective treatment for this disorder. MicroRNAs (miRNAs or miRs) have been increasingly shown to be associated with age-related diseases and are emerging as promising therapeutic targets. In this study, we investigated whether miR-29b is involved in the degeneration of cochlear hair cells. To examine our hypothesis, nuclear staining and terminal deoxynucleotidyl transferase dUTP nick-end labeling (TUNEL) were used to quantify the hair cell counts. RT-qPCR and western blot analysis were used to examine miR-29b/sirtuin 1 (SIRT1)/proliferator-activated receptor-gamma coactivator $1 \alpha(\mathrm{PGC}-1 \alpha)$ signaling in cochlear hair cells. We found that there was a significant degeneration of cochlear hair cells and a higher expression of miR-29b in aged C57BL/6 mice compared with young mice. There was also an age-related decrease in the expression of SIRT1 and PGC-1 $\alpha$. In the inner ear cell line, HEI-OC1, miR-29b overexpression (by transfection with miR-29b mimic) inhibited SIRT1 and PGC-1 $\alpha$ expression, leading to an increase in mitochondrial dysfunction and apoptosis. Moreover, the inhibition of miR-29b (by transfection with miR-29b inhibitor) increased SIRT1 and PGC-1 $\alpha$ expression, while it decreased apoptosis. Taken together, our findings support a link between age-related cochlear hair cell apoptosis and miR-29b/SIRT1/PGC-1 $\alpha$ signaling, which may present an attractive pharmacological target for the development of novel drugs for the treatment of age-related hearing loss.
\end{abstract}

Correspondence to: Dr Tao Xue or Dr Ding-Jun Zha, Department of Otolaryngology, Xijing Hospital, The Fourth Military Medical University, Changle West Street 127, Xi'an, Shaanxi 710032, P.R. China E-mail: taoxuexa@163.com

E-mail: dingjun_zha@163.com

${ }^{*}$ Contributed equally

Key words: microRNA-29b, cochlear hair cells, apoptosis, sirtuin 1/ peroxisome proliferator-activated receptor-gamma coactivator $1 \alpha$, age-related hearing loss

\section{Introduction}

Age-related hearing loss (AHL), also known as presbycusis, is the most common form of hearing loss and the predominant agerelated neurodegenerative disease affecting approximately $40 \%$ of individuals by the age of 65 years (1). Thus far, there is no effective treatment available for AHL. The irreversible loss of cochlear hair cells in the inner ear is one of the main causes of AHL in both aging humans and in animal models (2-4), and decreasing the loss of cochlear hair cells may present an attractive therapeutic strategy for the treatment of AHL.

MicroRNAs (microRNAs or miRs) are non-coding RNAs, 18-25 nucleotides in length, which regulate the expression of target mRNAs, as well as influence cellular senescence and aging (5-7). Recently, alterations in the expression of the miR-29 family and the miR-34 family have been documented during aging in the mammalian inner ear $(8,9)$. Of interest is that miR-29b has been shown to be involved in cellular senescence and the apoptosis of nerve cell lines, the brain and the liver during aging $(10,11)$. However, the functions of miR-29b regarding apoptosis are not yet fully understood. Sirtuin 1 (SIRT1) is a nicotinamide adenine dinucleotide (NAD)dependent deacetylase that acts as a sensor to regulate the intracellular oxidative stress status by the deacetylation of its substrates, including proliferator-activated receptor-gamma coactivator $1 \alpha$ (PGC-1 $\alpha)$, a transcriptional coregulator that binds to numerous transcription factors to promote mitochondrial biogenesis and oxidative metabolism (12-14). Oxidative stress, which is mainly caused by mitochondrial dysfunction, is known to play a causal role in AHL through the induction of apoptosis $(15,16)$. Of note, SIRT1 has been confirmed to be a direct target of miR-29b (17).

In this study, we hypothesized that miR-29b/SIRT1/PGC-1 $\alpha$ signaling may play a role in hair cell death and AHL pathogenesis, and that strategies aimed at inhibiting miR-29b activity or restoring SIRT1 function, may prove to be be beneficial in the treatment of AHL. To test our hypothesis, cochlear miR-29b/ SIRT1/PGC-1 $\alpha$ expression was examined in C57BL/6 mice, a mouse model of AHL. Additionally, the potential effects of miR-29b on the expression of SIRT1 and PGC- $1 \alpha$ and the underlying mechanisms were assessed using HEI-OC1 inner ear cells. 


\section{Materials and methods}

Animals and groups. Sixty C57BL/6 mice were procured from the Laboratory Animal Center of the Fourth Military Medical University (Xian, China) and divided into 2 groups as follows: a 'young' group (1-2 months of age, 30 mice) and an 'old' group (12-16 months of age, 30 mice), and were fed standard chow. Hearing tests were conducted on all mice, and cochlear tissues were collected for hair cell counting. All procedures involving animals were conducted in accordance with the Guidelines for Animal Experiments approved by the Ethics Committee for Animal Studies of the Fourth Military Medical University.

Auditory brainstem response (ABR). All mice were anesthetized with an intraperitoneal injection mixture that contained $100 \mathrm{mg} / \mathrm{kg}$ ketamine and $10 \mathrm{mg} / \mathrm{kg}$ xylazine. ABR measurements were performed by inserting subdermal needle electrodes at the vertex (active), under the left ear (reference), and under the right ear (ground). Tucker-Davis Technologies (TDT System III, Alachua, FL, USA) hardware and software were used to generate acoustic signals and to process the responses. Ten-millisecond (msec) tone bursts with a $1 \mathrm{msec}$ rise or fall time were presented at $4,8,16$ and $32 \mathrm{kHz}$ at a rate of $21.1 / \mathrm{sec}$. The average response to 1,000 stimuli was obtained by reducing the sound intensity at $5 \mathrm{~dB}$ intervals near the threshold, which was defined as the lowest stimulation decibel level at which a positive wave in the evoked response trace was evident.

Tissue preparation and isolation of cochlear tissue mitochondria. After the ABR recordings, the mice were decapitated, and the cochleae were removed and fixed by immersion in $4 \%$ paraformaldehyde in $0.1 \mathrm{mM}$ phosphate-buffered saline (PBS, pH 7.4) overnight at $4^{\circ} \mathrm{C}$, followed by being decalcified in $4 \%$ sodium ethylenediaminetetraacetic acid for 4 days. Following incubation in $30 \%$ sucrose overnight, the cochleae were cut into $10-\mathrm{mm}$ thick slices and stored at $-20^{\circ} \mathrm{C}$. For RNA and protein preparations, the cochlear tissues were dissected with small forceps, snap-frozen in liquid nitrogen and stored at $-80^{\circ} \mathrm{C}$. The mitochondria were isolated from the cochleae using differential centrifugation and TOM22 immunomagnetic affinity isolation. Briefly, the cochleae were dounce homogenized in isolation medium (IM) containing $225 \mathrm{mM}$ sucrose, $75 \mathrm{mM}$ mannitol, $1 \mathrm{mM}$ EGTA, $5 \mathrm{mM}$ HEPES, and complete Mini, EDTA-free protease inhibitor cocktail (Roche Diagnostics, Indianapolis, IN, USA) adjusted to $\mathrm{pH}$ 7.4. The homogenate was centrifuged at $1,000 \mathrm{x}$ g for $10 \mathrm{~min}$, the supernatant collected and the pellet was resuspended in IM. Following a second centrifugation at $1,000 \mathrm{x}$ for $10 \mathrm{~min}$, the pooled supernatants were centrifuged at $8,000 \mathrm{x}$ for $10 \mathrm{~min}$. The pellet containing the mitochondria was further purified using anti-TOM22 immunomagnetic affinity isolation (Miltenyi Biotech, Bergisch Gladbach, Germany). Purified mitochondria were lysed in $100 \mathrm{mM}$ Tris- $\mathrm{HCl}$ with $4 \%(\mathrm{w} / \mathrm{v})$ SDS and 0.1 M DTT adjusted to $\mathrm{pH} 7.6$ using brief sonication and incubation at $95^{\circ} \mathrm{C}$ for $5 \mathrm{~min}$.

Nuclear staining and terminal deoxynucleotidyl transferase dUTP nick-end labeling (TUNEL) assay. Following fixation with $4 \%$ paraformaldehyde, the organ of Corti, which was isloated from the cochleae under a dissecting microscope, was rinsed with PBS, immersed in $0.5 \%$ Triton X-100 for $15 \mathrm{~min}$ at room temperature, incubated with 4',6-diamidino-2-phenylindole (DAPI, $10 \mathrm{mg} / \mathrm{ml}$; Sigma, St. Louis, MO, USA) for $10 \mathrm{~min}$, and mounted on glass slides in 50\% glycerol. Some surface preparations of the organ of Corti were incubated with $100 \mathrm{ml}$ of TUNEL reaction mixture (Roche Diagnostics) containing dUTP-FITC and terminal deoxynucleotidyl transferase at $37^{\circ} \mathrm{C}$ in the dark for $60 \mathrm{~min}$, followed by incubation with propidium iodide (PI) staining solution (10 mg/ml PI in PBS) for $30 \mathrm{~min}$ at room temperature. The samples were observed and imaged using an Olympus BX63 microscope (Olympus, Ishikawa, Japan). Hair cell counts were identified by the presence of a nucleus and performed in two locations: at 10-20\% (outer hair cells) and $65-70 \%$ (inner hair cells) of the total cochlear duct distance from the apex, which approximately corresponds to the frequency range of $7-8$ or $32-36 \mathrm{kHz}$ (18). Hair cells were counted in 6 mice from each group and were considered to be degenerated if the cell nuclei were absent.

HEI-OC1 cell culture and cell groups. The HEI-OC1 cells were a gift from Dr. Federico Kalinec (House Ear Institute, Los Angeles, CA, USA) and were cultured in Dulbecco's modified Eagle's medium supplemented with 10\% fetal bovine serum (both from Gibco, Gaithersburg, MD, USA) at $33^{\circ} \mathrm{C}$ and $7 \% \mathrm{CO}_{2}$. The cells were divided into 7 different groups as follows: the control group, the $\mathrm{H}_{2} \mathrm{O}_{2}$ group and 5 transfection groups. The cells in the transfection groups were transfected with a miR-29b mimic, a negative control (pre-miRNA), or a miR-29b inhibitor (GenePharma, Shanghai, China). Fulllength SIRT1 was amplified by PCR using cDNA, and was confirmed by sequencing and subcloning into a plk0.1 expression vector (Invitrogen, Carlsbad, CA, USA). The HEI-OC1 cells were placed in a 6-well plate and transfected with plk0.1-scramble or plk0.1-SIRT1 using Lipofectamine 2000 (Invitrogen). Following transfection, the HEI-OC1 cells were exposed to $50 \mu \mathrm{M} \mathrm{H}_{2} \mathrm{O}_{2}$ for $1 \mathrm{~h}$.

Measurement of mitochondrial membrane potential. The mitochondrial dye, 5,5',6,6'-tetrachloro-1,1',3,3'-tetramethylbenzimidazolylcarbocyanine iodide (JC-1), was used to determine the mitochondrial membrane potential. JC-1 exhibits potential-dependent accumulation in the mitochondria, which is indicated by a fluorescence emission shift from green $(530 \mathrm{~nm})$ to red $(590 \mathrm{~nm})$. Accordingly, mitochondrial depolarization is indicated by a decrease in the red/green fluorescence intensity ratio. In brief, $\mathrm{HEI}-\mathrm{OC} 1$ cells were cultured in a 24 -well plate and subjected to the different treatments as described above, followed by incubation with $10 \mathrm{mg} / \mathrm{ml} \mathrm{JC}-1$ for $30 \mathrm{~min}$ at $37^{\circ} \mathrm{C}$. The cells were then scanned using a FlexStation 3 multi-mode microplate reader (Molecular Devices, Silicon Valley, USA). The fluorescence ratio (590 to $530 \mathrm{~nm}$ ) was used for quantitative analysis.

Cell proliferation assay. After the concentration was adjusted to $2 \times 10^{6}$ cells/well, the cells were seeded in 96 -well plates and then stained at the indicated time points with $100 \mu 1$ sterile MTT (Molecular Probes, Eugene, OR, USA) for $4 \mathrm{~h}$ at $37^{\circ} \mathrm{C}$. The supernatant was removed and $200 \mu 1$ dimethyl sulfoxide (DMSO) (Invitrogen) was added for $30 \mathrm{~min}$ to dissolve the formazan crystals. The spectrometric absorbance at a wavelength of $490 \mathrm{~nm}$ was measured on a SpectraMax M5 
microplate reader (Molecular Devices). Each sample was tested in triplicate and all experiments were performed 3 times.

Apoptosis assay. Flow cytometric analysis with Annexin V-FITC/PI double staining was performed using an Annexin V-FITC apoptosis detection kit (Bio-Rad, Hercules, CA, USA) to measure cell apoptosis. All groups of HEI-OC1 cells were incubated for $72 \mathrm{~h}$, and the cells were then harvested and centrifuged at 1,200 x $\mathrm{g}$ for $5 \mathrm{~min}$, followed by washing twice with ice-cold PBS. Following the addition of $5 \mu \mathrm{l}$ of Annexin V, $5 \mu \mathrm{l}$ of PI and $500 \mu \mathrm{l}$ of PBS to the cells, the cells were incubated at room temperature for $15 \mathrm{~min}$ in the dark. Cell apoptosis was analyzed on a FACS can flow cytometer (CyAn ADP; Beckman Coulter, Brea, CA, USA). Annexin V-FITC positive and PI-negative cells were scored as apoptotic. Double-stained cells were considered as either necrotic or late apoptotic cells.

Reverse transcription-quantitative (real-time) polymerase chain reaction $(R T-q P C R)$. Total RNA was isolated from the cells using TRIzol Reagent (Invitrogen) according to the manufacturer's instructions, and $1 \mathrm{mg}$ total RNA was reverse transcribed using the ReverTra-Plus ${ }^{\mathrm{TM}}$ kit (Toyobo, Osaka, Japan). The primer sequences used for amplification were as follows: SIRT1 forward, 5'-AAAGGAATTGGTTCATTTATCAGAG-3' and reverse, 5'-TTGTGGTTTTTCTTCCACACA-3'; PGC-1 $\alpha$ forward, 5'-AAACTTGCTAGCGGTCCTCA-3' and reverse, 5'-TGGCTGGTGCCAGTAAGAG-3'; and $\beta$-actin forward, 5'-TGAACGGGAAGCTCACTGG-3' and reverse, 5'-GCTTCA CCACCTTCTTGATGTC-3'. Complementary DNA samples were amplified using SYBR Premix Ex Taq (Tli RNaseH Plus; Takara, Otsu, Japan) and detected with the Roche LightCycler 480 real-time PCR system. $\beta$-actin was used as an internal control for SIRT1 and PGC-1 $\alpha$ normalization.

For the analysis of miR-29b expression, enriched small RNAs were isolated from the cochlear tissues using TRIzol reagent, with $500 \mathrm{ng}$ of RNA being reverse transcribed using specific miRNA stem-loop primers and a PrimeScript RT reagent kit (Takara). Mature miRNA expression was measured with Takara Taq version 2.0 plus dye (Takara) according to the manufacturer's instructions, and the miRNA levels were normalized to U6 small nuclear RNA expression.

Western blot analysis. The cochlear tissues and cultured HEI-OC1 cells were homogenized in ice-cold radioimmunoprecipitation assay lysis buffer (Santa Cruz Biotechnology, Inc., Santa Cruz, CA, USA) for $30 \mathrm{~min}$ and centrifuged at $12,000 \mathrm{x} \mathrm{g}$ at $4^{\circ} \mathrm{C}$ for $10 \mathrm{~min}$, and the supernatants were collected. Protein concentrations were determined using a protein assay dye reagent (Bio-Rad, Hercules, CA, USA). Protein samples (50 mg) were resolved by sodium dodecyl sulfate polyacrylamide gelelectrophoresis (SDS-PAGE), and proteins were transferred onto polyvinylidene fluoride membranes (Millipore, Billerica, MA, USA) and blocked with 5\% non-fat dry milk in PBS with $0.1 \%$ Tween-20 (PBS-T). The membranes were incubated with anti-SIRT1 (1:1,000; sc-74504) or anti-PGC-1 $\alpha(1: 1,000$; sc-13067) (both from Santa Cruz Biotechnology, Inc.) antibodies overnight, washed 3 times (10 min each) with PBS-T, and incubated with goat anti-mouse IgG (1:5,000; F020220, Sigma) for $1 \mathrm{~h}$. Following extensive washing of the membranes with $1 \mathrm{X}$ PBS-T, the immunoreactive bands were visualized by enhanced chemiluminescence (Millipore). Band intensities were quantified by densitometric analysis using NIH ImageJ software, and $\beta$-actin (1:2,000; sc-47778, Santa Cruz Biotechnology, Inc.) was used as a loading and an internal control to enable sample normalization.

Statistical analysis. All experiments were independently repeated at least 3 times. One-way analysis of variance (ANOVA) with Fisher's post hoc test and Student's t-test was used for statistical analysis. Values of $\mathrm{p}<0.05$ were considered to indicate statistically significant differences.

\section{Results}

Progressive hearing threshold shifts and hair cell loss. In an attempt to elucidate the mechanisms responsible for the progression of AHL, hearing function in young and aged C57BL/6 mice was evaluated by ABR, which is an objective electrophysiological test to determine hearing function. The average thresholds from the aged mice $(78 \pm 9 \mathrm{~dB}$ at $4 \mathrm{kHz}, 67 \pm 10 \mathrm{~dB}$ at $8 \mathrm{kHz}, 89 \pm 8 \mathrm{~dB}$ at $16 \mathrm{kHz}$ and $110+6 \mathrm{~dB}$ at $32 \mathrm{kHz}$ ) were significantly elevated compared with those from the young mice $(45 \pm 8 \mathrm{~dB}$ at $4 \mathrm{kHz}, 32 \pm 10 \mathrm{~dB}$ at $8 \mathrm{kHz}, 48 \pm 8 \mathrm{~dB}$ at $16 \mathrm{kHz}$ and $59 \pm 6 \mathrm{~dB}$ at $32 \mathrm{kHz}$ ) at all tested frequencies, indicating that the aged C57BL/6 mice developed significant hearing loss (Fig. 1A). After the ABR measurements, the cochleae were processed for surface preparations and quantitative hair cell counts. The percentage inner hair cells and outer hair cells in the aged mice was significantly decreased compared with the young mice in the apical cochlear turn (Fig. 1B and C).

Mitochondrial dysfunction induced by aging in the C57BL/6 mice. To confirm whether mitochondrial dysfunction is involved during aging, the mitochondrial dye, JC-1, was used to determine the mitochondrial membrane potential in the cochleae. A significant decrease in mitochondrial membrane potential was observed in thecochleae of the aged C57BL/6 mice compared with the young ones, indicating the appearance of mitochondrial dysfunction in the aged C57BL/6 mice (Fig. 1D).

Expression of miR-29b, SIRT1 and PGC-1 $\alpha$ in the cochleae during aging. To further elucidate the mechanisms responsible for hearing loss, RT-qPCR was used to examine the miR-29b, SIRT1 and PGC-1 $\alpha$ mRNA expression levels in the cochleae of young and aged C57BL/6 mice, while the protein expression levels of SIRT1 and PGC-1 $\alpha$ were examined by western blot analysis. The results revealed that miR-29b expression was significantly upregulated in the aged C57BL/6 mice compared with the young mice (Fig. 2). Conversely, the mRNA and protein expression levels of SIRT1 and PGC-1 $\alpha$ were markedly decreased in the cochleae of the aged mice.

Effect of $m i R-29 b$ on the apoptosis and proliferation of $\mathrm{HEI}-\mathrm{OCl}$ cells. It is already known that $\mathrm{H}_{2} \mathrm{O}_{2}$-induced oxidative stress promotes cell apoptosis (19). In this study, to examine the effects of miR-29b on cell survival, HEI-OC1 cells transfected with the miR-29b mimic or miR-29b inhibitor were exposed to $50 \mu \mathrm{M} \mathrm{H}_{2} \mathrm{O}_{2}$ for $1 \mathrm{~h}$, and then apoptosis was measured by flow cytometric analysis. The results revealed that the overexpres- 


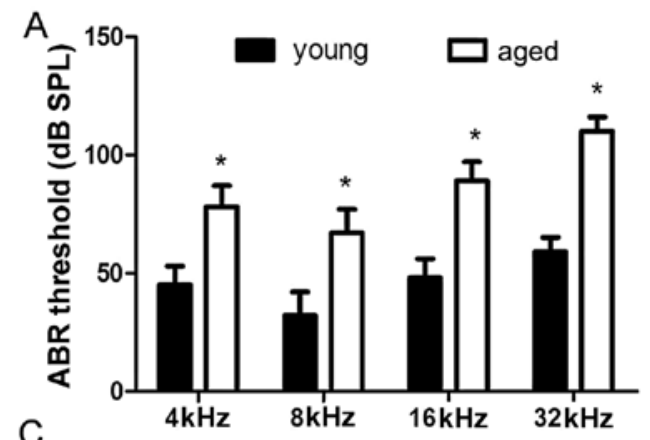

B
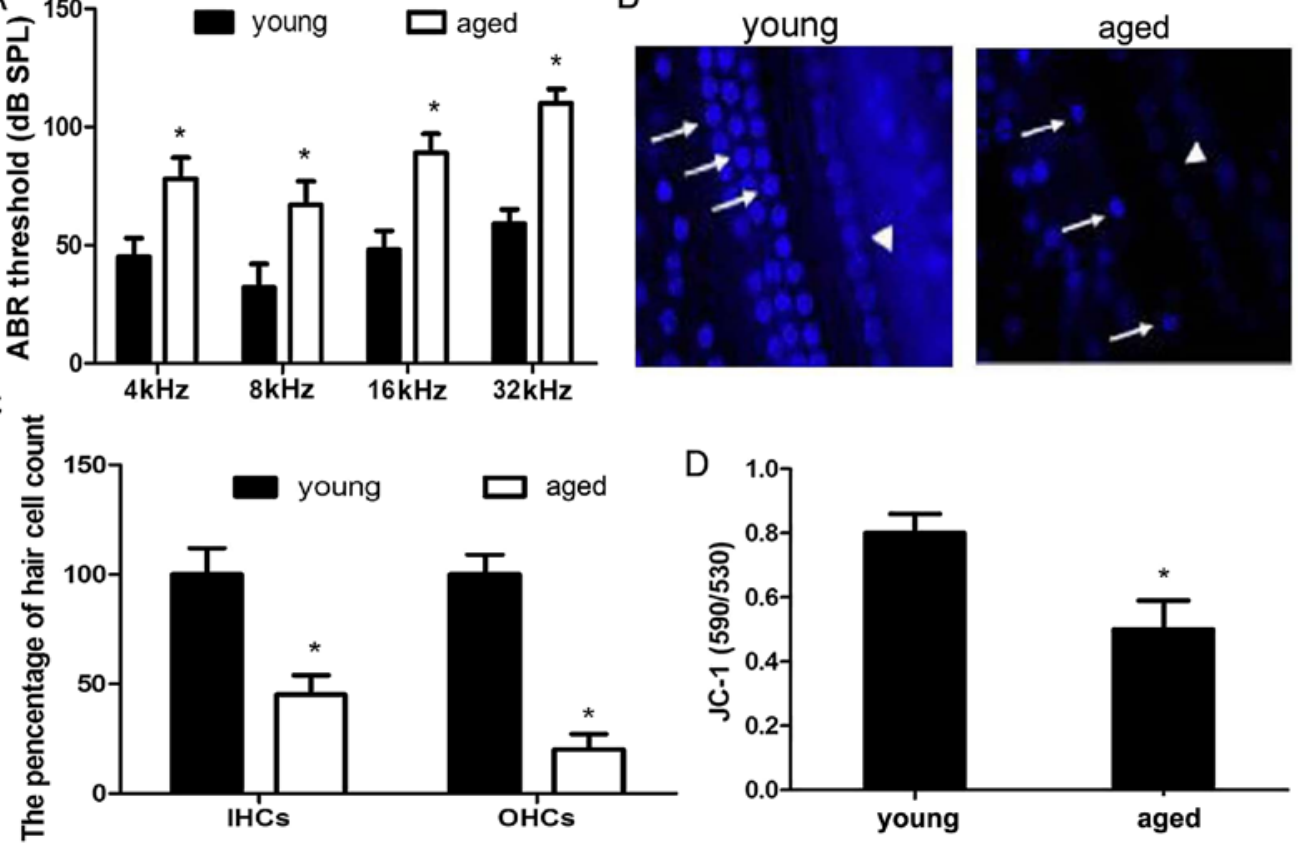

Figure 1. Increased auditory brainstem response thresholds, hair cell loss and mitochondrial dysfunction in aged C57BL/6 mice. (A) Auditory brainstem response (ABR) measurements were performed in C57BL/6 mice aged 1-2 months (young) and 12-16 months (aged) at 4, 8, 16 and $32 \mathrm{kHz}$. " p<0.05 compared to the young group. (B and C) Hair cell loss was observed in C57BL/6 mice at 12-16 months of age. Outer hair cells (OHCs) are indicated by arrows and inner hair cells (IHCs) are indicated by arrowheads. (D) Severe mitochondrial dysfunction was observed in C57BL/6 mice at 12-16 months of age. " $\mathrm{p}<0.05$ compared to the young group.

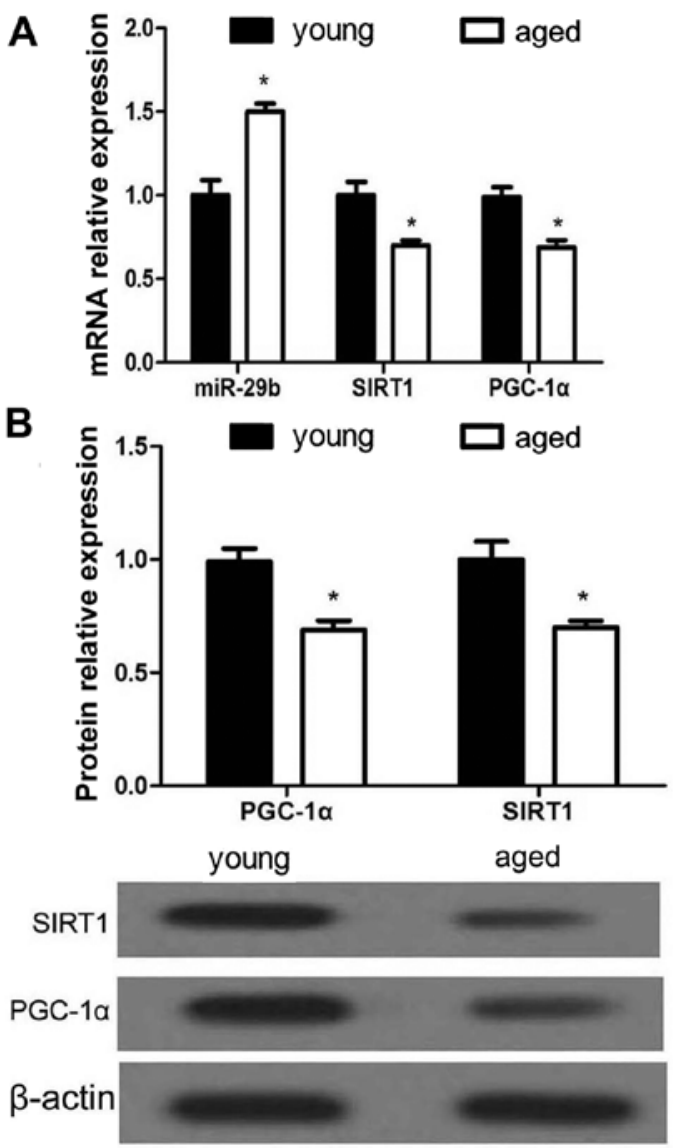

Figure 2. The expression of miR-29b, sirtuin 1 (SIRT1) and proliferatoractivated receptor-gamma coactivator $1 \alpha$ (PGC- $1 \alpha)$ in the cochleae of young and aged C57BL/6 mice. (A) RT-qPCR analysis of miR-29b, SIRT1 and PGC- $1 \alpha$ in young and aged C57BL/6 mice. "p $<0.05$, compared to the young mice. (B) Western blot analysis of SIRT1 and PGC-1 $\alpha$ in young and aged C57BL/6 mice. " $\mathrm{p}<0.05$, compared to the young mice. sion of miR-29b promoted cell apoptosis, whereas transfection with the miR-29b inhibitor decreased apoptosis compared with the $\mathrm{H}_{2} \mathrm{O}_{2}$ group (Fig. 3A and B). The results of MTT assay demonstrated that transfection with the miR-29b mimic significantly suppressed HEI-OC1 cell proliferation compared with the $\mathrm{H}_{2} \mathrm{O}_{2}$ group, whereas transfection with the miR-29b inhibitor promoted cell proliferation (Fig. 3C).

miR-29b overexpression induces mitochondrial dysfunction. To determine whether miR-29b is involved in mitochondrial dysfunction, the mitochondrial membrane potential was determined using the mitochondrial dye, JC-1, in all groups of HEI-OC1 cells. Transfection of the cells with the miR-29b mimic exacerbated the decrease in $\mathrm{H}_{2} \mathrm{O}_{2}$-induced mitochondrial membrane potential observed in the $\mathrm{H}_{2} \mathrm{O}_{2}$ group (Fig. 3D). By contrast, transfection with the miR-29b inhibitor attenuated the $\mathrm{H}_{2} \mathrm{O}_{2}$-induced mitochondrial dysfunction. These results indicate that the overexpression of miR-29b induces mitochondrial dysfunction.

miR-29b modulates the expression of SIRT1 and PGC-1 $\alpha$ in HEI-OCl cells. To test the hypothesis that miR-29b modulates SIRT1 and PGC-1 $\alpha$ expression, the mRNA and protein expression levels of SIRT1 and PGC- $1 \alpha$ were measured by RT-qPCR and western blot analysis, respectively in the HEI-OC1 cells following transfection with the miR-29b mimic or the miR-29b inhibitor. The overexpression of miR-29b suppressed the mRNA and protein expression of SIRT1 and PGC-1 $\alpha$ compared with the $\mathrm{H}_{2} \mathrm{O}_{2}$ group, whereas transfection with the miR-29b inhibitor promoted the expression of SIRT1 and PGC-1 $\alpha$ (Fig. 4). These findings suggest that miR-29b modulates the expression of SIRT1 and PGC-1 $\alpha$ in HEI-OC1 cells. 
A
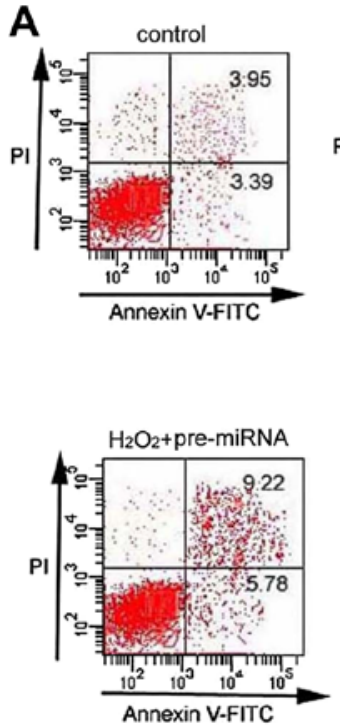

$\mathrm{H}_{2} \mathrm{O}_{2}+$ miR-29b inhibitor

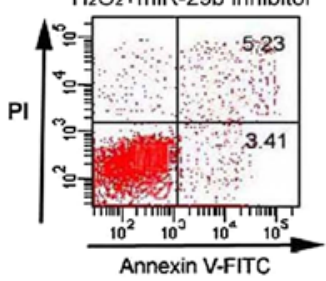

B
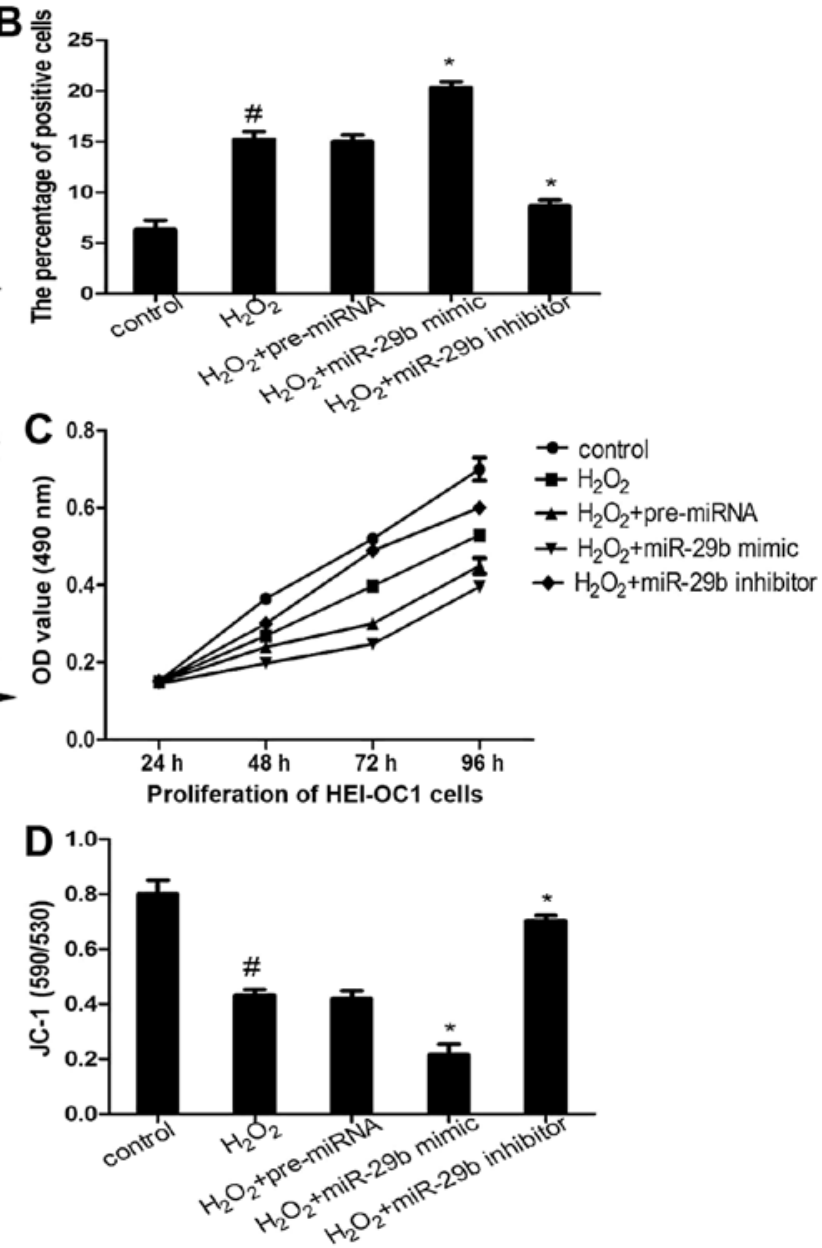

Figure 3. miR-29b promotes HEI-OC1 cell apoptosis and mitochondrial dysfunction. (A and B) HEI-OC1 cell apoptosis were measured using Annexin V and propidium iodine (PI) staining. (C) The proliferation of HEI-OC1 cells was measured by MTT assay. (D) The mitochondrial membrane potential of HEI-OC1 cells subjected to different treatments was measured using the mitochondrial dye, JC-1. " $\mathrm{p}<0.05$ compared to the control group; ${ }^{*} \mathrm{p}<0.05$ compared to the $\mathrm{H}_{2} \mathrm{O}_{2}$ group.

\section{A}

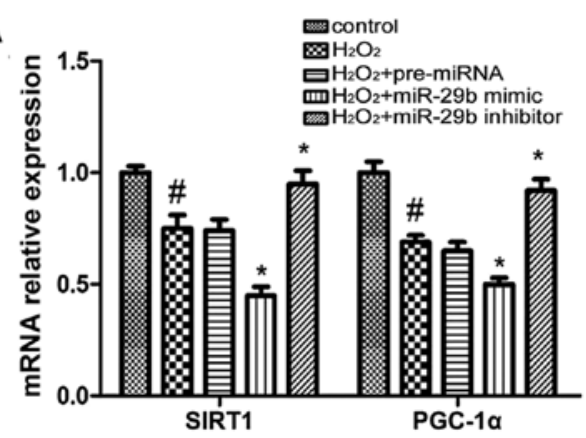

B
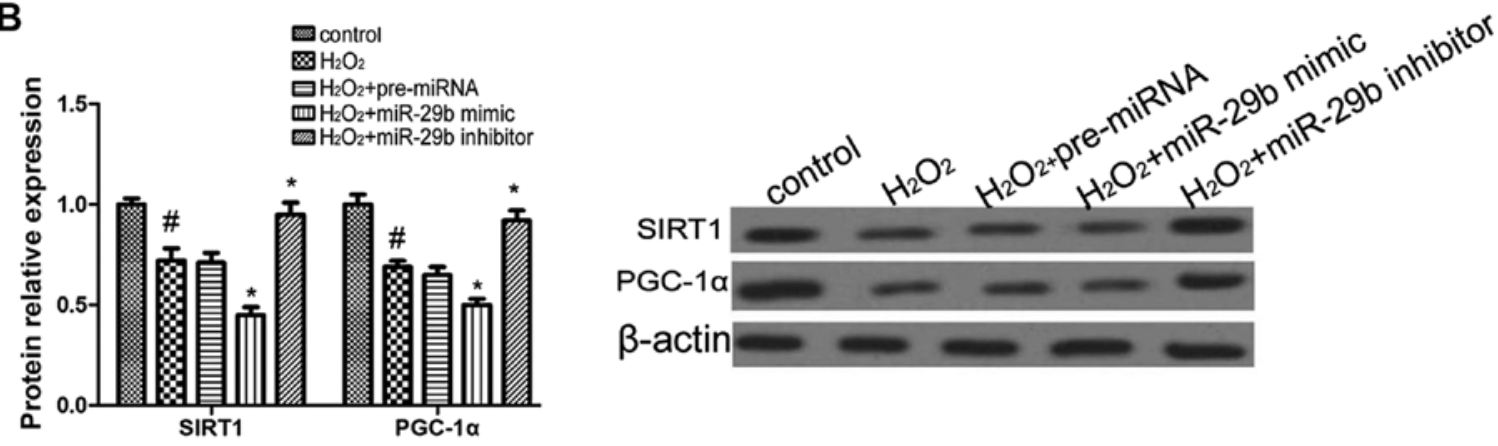

Figure 4. miR-29b regulates the expression of sirtuin 1 (SIRT1) and proliferator-activated receptor-gamma coactivator $1 \alpha$ (PGC-1 $\alpha$ ) in HEI-OC1 cells (A) RT-qPCR analysis of SIRT1 and PGC- $1 \alpha$ in HEI-OC1 cells subjected to different treatments. " $\mathrm{p}<0.05$ compared to the control group; ${ }^{\mathrm{p}}<0.05$ compared to the $\mathrm{H}_{2} \mathrm{O}_{2}$ group. (B) Protein expression of SIRT1 and PGC-1 $\alpha$ determined by western blot analysis in HEI-OC1 cells subjected to different treatments. ${ }^{\sharp} \mathrm{p}<0.05$ compared to the control group; ${ }^{*} \mathrm{p}<0.05$ compared to the $\mathrm{H}_{2} \mathrm{O}_{2}$ group. 

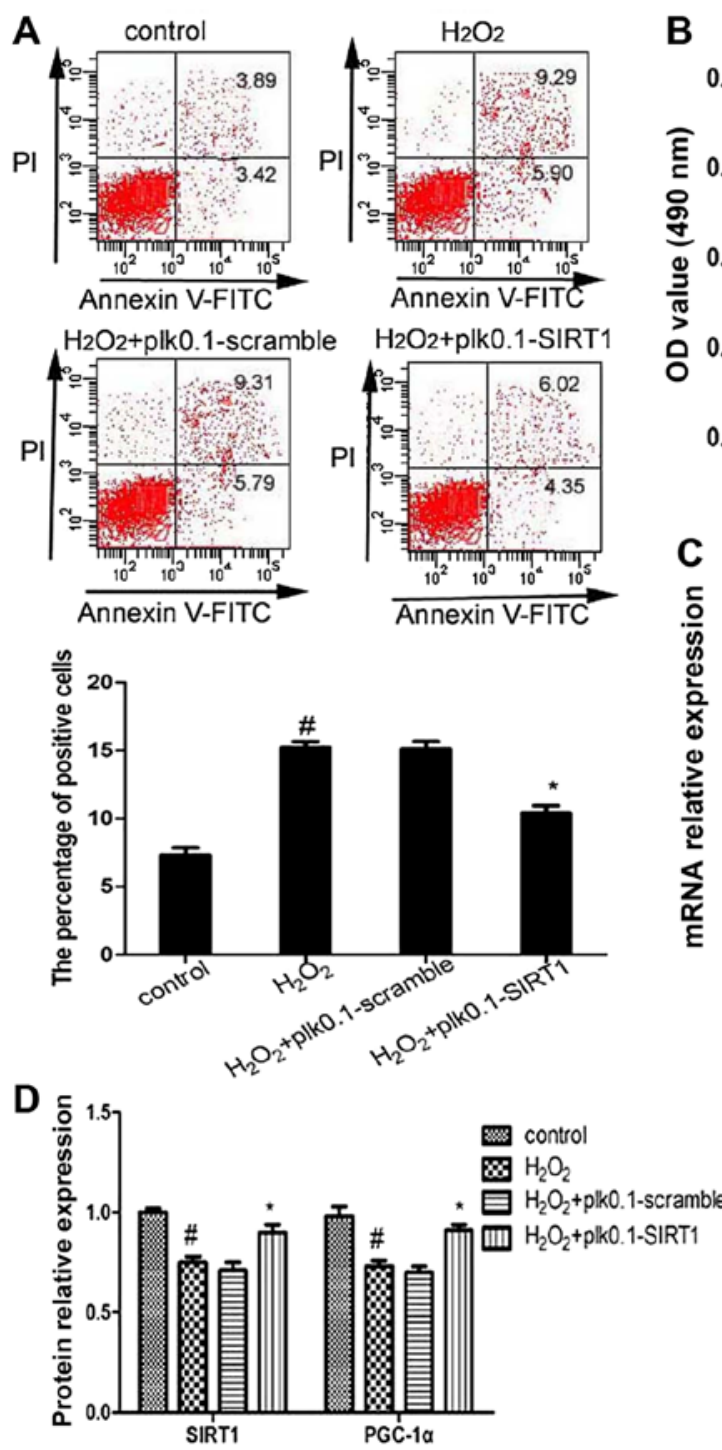

C
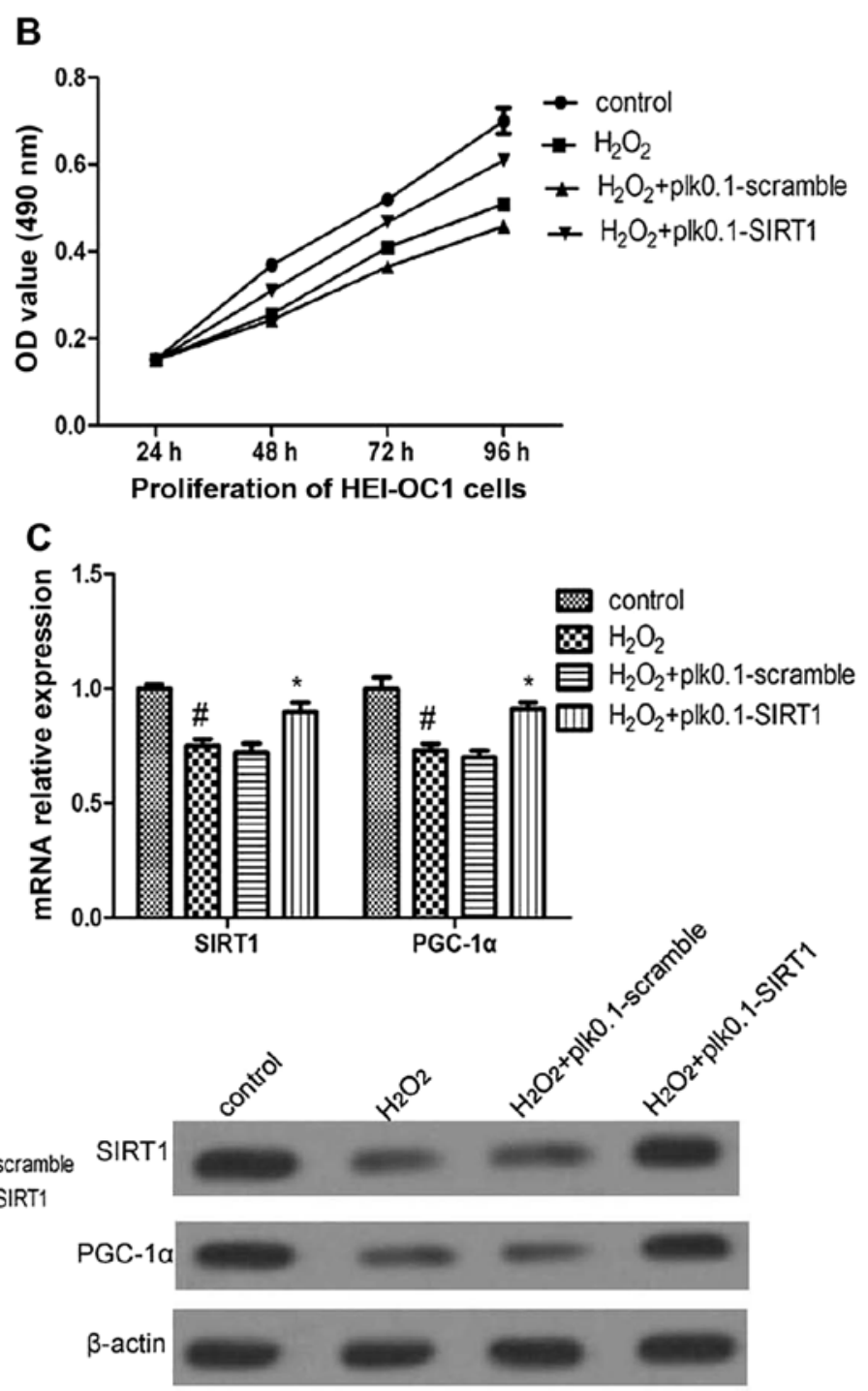

Figure 5. Sirtuin 1 (SIRT1) regulates the expression of proliferator-activated receptor-gamma coactivator $1 \alpha$ (PGC-1 $\alpha$ ), as well as the apoptosis and proliferation of HEI-OC1 cells. (A) HEI-OC1 cell apoptosis was measured by Annexin V and propidium iodine staining. (B) The proliferation of HEI-OC1 cells was measured by MTT assay. (C) RT-qPCR analysis of SIRT1 and PGC-1 $\alpha$ in HEI-OC1 cells subjected to different treatments. ${ }^{\# p<0.05 ~ c o m p a r e d ~ t o ~ t h e ~ c o n t r o l ~ g r o u p ; ~}$ ${ }^{*} \mathrm{p}<0.05$ compared to the $\mathrm{H}_{2} \mathrm{O}_{2}$ group. (D) Protein expression analysis of SIRT1 and PGC-1 $\alpha$ determined by western blot analysis in HEI-OC1 cells subjected to different treatments. ${ }^{\#} \mathrm{p}<0.05$ compared to the control group; ${ }^{*} \mathrm{p}<0.05$ compared to the $\mathrm{H}_{2} \mathrm{O}_{2}$ group.

Effect of SIRT1 overexpression on PGC-1 $\alpha$ expression, as well as on HEI-OC1 cell apoptosis and proliferation. To examine the effects of SIRT1 on PGC-1 $\alpha$ expression, as well as on HEI-OC1 cell apoptosis and proliferation, the HEI-OC1 cells were transfected with plk0.1-scramble or plk-0.1-SIRT1 using Lipofectamine 2000 in DMEM.

The overexpression of SIRT1 significantly suppressed cell apoptosis and promoted cell proliferation, and also promoted the mRNA level and protein expression of PGC-1 $\alpha$ compared with the $\mathrm{H}_{2} \mathrm{O}_{2}$ group (Fig. 5). These results suggest that SIRT1 modulates the expression of PGC-1 $\alpha$, as well as the apoptosis and proliferation of HEI-OC1 cells.

\section{Discussion}

Aging is one of the most prominent factors which influence the onset and progression of multiple diseases, along with mitochondrial dysfunction, which is induced by oxidative stress. AHL, a common age-related disease, often occurs in those aged 65 years and above. A previous study demonstrated that the expression of miR-29b was upregulated in AHL (5). In the present study, our results also confirmed that miR-29b was overexpressed in the cochleae of aging C57BL/6 mice, which is the most widely used mouse model for the study of aging and age-associated diseases (20). Of note, in our study, there was a significant decrease in the counts of hair cells and increased mitochondrial dysfunction in the cochleae of the aged C57BL/6 mice, which is in agreement with the findings of previous studies indicating that hair cell apoptosis is a key contributor to the development of AHL $(4,21)$. SIRT1-mediated PCG-1 $\alpha$ deacetylation is necessary for the activation of mitochondrial fatty acid oxidation genes (22). In this study, an age-related decrease in SIRT1 and PGC-1 $\alpha$ expression along with mitochondrial dysfunction was noted in the cochleae of 
aged mice. Of note, SIRT1 has been confirmed to be a direct target of miR-29b (23). Thus, we hypothesized that miR-29b/ SIRT1/PGC-1 $\alpha$ signaling may play a crucial role during hair cell death and in the pathogenesis of AHL.

HEI-OC1, a conditionally immortalized mouse cell line derived from the postnatal organ of Corti, has been extensively used to elucidate pathways of hair cell pathology $(24,25)$. In this study, HEI-OC1 cells were divided into different groups (control, $\mathrm{H}_{2} \mathrm{O}_{2}, \mathrm{H}_{2} \mathrm{O}_{2}+\operatorname{miR}-29 \mathrm{~b}$ mimic, $\mathrm{H}_{2} \mathrm{O}_{2}+$ premiRNA, $\mathrm{H}_{2} \mathrm{O}_{2}+$ miR-29b inhibitor). It is well known that oxidative stress plays a causal role in AHL through the induction of apoptosis $(4,26)$. In this study, MTT and apoptosis assays indicated that $\mathrm{H}_{2} \mathrm{O}_{2}$ significantly suppressed cell proliferation and promoted apoptosis compared to the control group. However, the overexpression of miR-29b enhanced the $\mathrm{H}_{2} \mathrm{O}_{2}$ mediated suppression of cell proliferation and the promoting effect on cell apoptosis, while the knockdown of miR-29b attenuated these effects. Analysis of mitochondrial membrane potential using the mitochondrial dye, JC-1, indicated that the overexpression of miR-29b significantly decreased mitochondrial membrane potential, which exacerbated $\mathrm{H}_{2} \mathrm{O}_{2}$-induced mitochondrial dysfunction. These results indicated that the overexpression of miR-29b promoted the loss of hair cells and mitochondrial dysfunction.

SIRT1 is recognized as a lifespan modulator and is implicated in preventing a number of age-related diseases $(27,28)$, such as cancer, Alzheimer's disease and type 2 diabetes $(29,30)$. As a NAD-dependent deacetylase, SIRT1 also interacts with PGC-1 $\alpha$, which plays a critical role in apoptosis, energy homeostasis, longevity and mitochondrial function $(31,32)$. In this study, in the cochleae of aged C57BL/6 mice, the expressions levels of SIRT1 and PGC-1 $\alpha$ were significantly decreased. A previous study demonstrated that SIRT1 was the direct target gene of miR-29 (18). Moreover, in this study, transfection of HEI-OC1 cells with miR-29b mimic suppressed the expression of SIRT1 and PGC-1 $\alpha$, while the knockdown of miR-29b significantly upregulated the mRNA level and protein expression of SIRT1 and PGC-1 $\alpha$. Furthermore, the overexpression of SIRT1 significantly increased the expression of PGC-1 $\alpha$ in HEI-OC1 cells and suppressed cell apoptosis, and promoted cell proliferation. Thus, it can be concluded that the overexpression of miR-29b induces mitochondrial dysfunction and hair cell apoptosis through the downregulation of the expression of SIRT1 and PGC-1 $\alpha$ during aging.

In conclusion, the findings of this study support the hypothesis that miR-29b/SIRT1/PGC-1 $\alpha$ signaling is involved in the development and progression of AHL. miR-29b appears to modulate mitochondrial dysfunction and apoptosis through SIRT1/PGC-1 $\alpha$ signaling in HEI-OC1 cells. Overall, miR-29b/ SIRT1/PGC-1 $\alpha$ signaling may present an attractive pharmacological target for the development of novel drugs for the treatment of AHL reatment.

\section{Acknowledgements}

The present study was supported by grants from the National Natural Science Foundation of China (no. 81271070, no. 81271077 and no. 81470695) and the Science and Technology Research and Development Program of Shaanxi Province (no. 2014SF2-08).

\section{References}

1. Xiong H, Pang J, Yang H, Dai M, Liu Y, Ou Y, Huang Q, Chen S, Zhang Z, Xu Y, et al: Activation of miR-34a/SIRT1/p53 signaling contributes to cochlear hair cell apoptosis: Implications for agerelated hearing loss. Neurobiol Aging 36: 1692-1701, 2015.

2. Yamasoba T, Lin FR, Someya S, Kashio A, Sakamoto T and Kondo K: Current concepts in age-related hearing loss: Epidemiology and mechanistic pathways. Hear Res 303: 30-38, 2013.

3. Kidd Iii AR and Bao J: Recent advances in the study of age-related hearing loss: A mini-review. Gerontology 58: 490-496, 2012.

4. Someya S, Xu J, Kondo K, Ding D, Salvi RJ, Yamasoba T, Rabinovitch PS, Weindruch R, Leeuwenburgh C, Tanokura M and Prolla TA: Age-related hearing loss in C57BL/6J mice is mediated by Bak-dependent mitochondrial apoptosis. Proc Natl Acad Sci USA 106: 19432-19437, 2009.

5. Zhang Q, Liu H, McGee J, Walsh EJ, Soukup GA and He DZ: Identifying microRNAs involved in degeneration of the organ of corti during age-related hearing loss. PLoS One 8: e62786, 2013.

6. Lee S, Choi E, Cha MJ, Park AJ, Yoon C and Hwang KC: Impact of miRNAs on cardiovascular aging. J Geriatr Cardiol 12: 569-574, 2015.

7. Olivieri F, Albertini MC, Orciani M, Ceka A, Cricca M, Procopio AD and Bonafè M: DNA damage response (DDR) and senescence: Shuttled inflamma-miRNAs on the stage of inflammaging. Oncotarget 6: 35509-35521, 2015.

8. Andl T and Botchkareva NV: MicroRNAs (miRNAs) in the control of HF development and cycling: The next frontiers in hair research. Exp Dermatol 24: 821-826, 2015.

9. Ushakov K, Rudnicki A and Avraham KB: MicroRNAs in sensorineural diseases of the ear. Front Mol Neurosci 6: 52, 2013.

10. Fenn AM, Smith KM, Lovett-Rache AE, Guerau-de-Arellano M, Whitacre CC and Godbout TP: Increased micro-RNA 29b in the aged brain correlates with the reduction of insulin-like growth factor-1 and fractalkine ligand. Neurobiol Aging 34:2748-2758, 2013.

11. Kamran F, Andrade AC, Nella AA, Clokie SJ, Rezvani G, Nilsson O, Baron J and Lui JC: Evidence That up-regulation of microRNA-29 contributes to postnatal body growth deceleration. Mol Endocrinol 29: 921-932, 2015.

12. Lee YH, Chen HY, Su LJ and Chueh PJ: Sirtuin 1 (SIRT1) deacetylase activity and $\mathrm{NAD}^{+} / \mathrm{NADH}$ ratio are imperative for capsaicin-mediated programmed cell death. J Agric Food Chem 63: 7361-7370, 2015.

13. Liu H, Sheng M, Liu Y, Wang P, Chen Y, Chen L, Wang W and Li B: Expression of SIRT1 and oxidative stress in diabetic dry eye. Int J Clin Exp Pathol 8: 7644-7653, 2015.

14. Tan M, Tang C, Zhang Y, Cheng Y, Cai L, Chen X, Gao Y, Deng Y and Pan M: SIRT1/PGC-1 $\alpha$ signaling protects hepatocytes against mitochondrial oxidative stress induced by bile acids. Free Radic Res 49: 935-945, 2015.

15. Kwon DN, Park WJ, Choi YJ, Gurunathan S and Kim JH: Oxidative stress and ROS metabolism via down-regulation of sirtuin 3 expression in Cmah-null mice affect hearing loss. Aging (Albany NY) 7: 579-594, 2015.

16. Someya S, Yu W, Hallows WC, Xu J, Vann JM, Leeuwenburgh C, Tanokura M, Denu JM and Prolla TA: Sirt3 mediates reduction of oxidative damage and prevention of age-related hearing loss under caloric restriction. Cell 143: 802-812, 2010.

17. Zhou L, Xu DY, Sha WG, Shen L, Lu GY, Yin X and Wang MJ: High glucose induces renal tubular epithelial injury via Sirt1/NF-kappaB/microR-29/Keap1 signal pathway. J Transl Med 13: 352, 2015.

18. Viberg A and Canlon B: The guide to plotting a cochleogram. Hear Res 197: 1-10, 2004

19. Wang G, Tang C, Yan G and Feng B: Gene expression profiling of $\mathrm{H} 9 \mathrm{c} 2$ cells subjected to $\mathrm{HO}$-induced apoptosis with/without AF-HF001. Biol Pharm Bull 39: 207-214, 2015.

20. Han X, Ge R, Xie G, Li P, Zhao X, Gao L, Zhang H, Wang O, Huang F and Han F: Caspase-mediated apoptosis in the cochleae contributes to the early onset of hearing loss in A/J mice. ASN Neuro 7: 7, 2015.

21. Op de Beeck K, Schacht $\mathbf{J}$ and Van Camp G: Apoptosis in acquired and genetic hearing impairment: The programmed death of the hair cell. Hear Res 281: 18-27, 2011.

22. McCarty MF, DiNicolantonio JJ and O'Keefe JH: Ketosis may promote brain macroautophagy by activating Sirtl and hypoxiainducible factor-1. Med Hypotheses 85: 631-639, 2015. 
23. Xu Z,Zhang L, Fei X, Yi X,Li W and Wang Q: The miR-29b-Sirt1 axis regulates self-renewal of mouse embryonic stem cells in response to reactive oxygen species. Cell Signal 26: 1500-1505, 2014.

24. Chen FQ, Zheng HW, Hill K and Sha SH: Traumatic noise activates Rho-family GTPases through transient cellular energy depletion. J Neurosci 32: 12421-12430, 2012.

25. Kim HJ, Lee JH, Kim SJ, Oh GS, Moon HD, Kwon KB, Park C, Park BH, Lee HK, Chung SY, et al: Roles of NADPH oxidases in cisplatin-induced reactive oxygen species generation and ototoxicity. J Neurosci 30: 3933-3946, 2010.

26. Jiang H, Talaska AE, Schacht J and Sha SH: Oxidative imbalance in the aging inner ear. Neurobiol Aging 28: 1605-1612, 2007.

27. Zeng Y and Yang K: Sirtuin 1 participates in the process of age-related retinal degeneration. Biochem Biophys Res Commun 468: 167-172, 2015.
28. Maiese K: SIRT1 and stem cells: In the forefront with cardiovascular disease, neurodegeneration and cancer. World J Stem Cells 7: 235-242, 2015.

29. Herskovits AZ and Guarente L: SIRT1 in neurodevelopment and brain senescence. Neuron 81: 471-483, 2014.

30. Sebastián C, Satterstrom FK, Haigis MC and Mostoslavsky R: From sirtuin biology to human diseases: An update. J Biol Chem 287: 42444-42452, 2012.

31. Seo S, Lee MS, Chang E, Shin Y, Oh S, Kim IH and Kim Y: Rutin increases muscle mitochondrial biogenesis with AMPK activation in high-fat diet-induced obese rats. Nutrients 7: 8152-8169, 2015.

32. Gerhart-Hines Z, Rodgers JT, Bare O, Lerin C, Kim SH, Mostoslavsky R, Alt FW, Wu Z and Puigserver P: Metabolic control of muscle mitochondrial function and fatty acid oxidation through SIRT1/PGC-1alpha. EMBO J 26: 1913-1923, 2007. 